\title{
Chemische Untersuchungen über die Inhaltsstoffe von Pteris cretica L.
}

In den vorigen Mitteilungen ${ }^{\mathbf{1}, 2)}$ haben wir über die Isolierung einiger Diterpen-Verbindungen aus den Rhizomen von Pteris cretica L. (Pteridaceae) und deren Struktur-Aufklärung berichtet. Wir haben nun die Struktur eines weiteren Diterpenalkohols (als Substanz C bezeichnet) aufgeklärt.

Substanz C. (I), $\mathrm{C}_{20} \mathrm{H}_{34} \mathrm{O}_{3}$, wurde als farblose Prismen vom Schmp. $235-236^{\circ}$ sowie $[\alpha]_{\mathrm{D}}^{25}$ : $-25^{\circ}\left(c=0.18\right.$, Äthanol) erhalten. Das Infrarot (IR)-Spektrum ${ }^{3)}$ zeigt die Absorbtionsbande der Hydroxylgruppen bei $3300 \mathrm{~cm}^{-\mathbf{1}}$. (I) gab bei der Acetylierung mit Essigsäureanhydrid in Pyridin ein Diacetat (II) $\mathrm{C}_{24} \mathrm{H}_{38} \mathrm{O}_{5}$, Schmp. 216-219 mit NMR (Kernmagnetische Resonanz)-Signalen ${ }^{3)}$ bei 1.97, 2.00 ppm für zwei Acetylgruppen und IR-Banden bei 3450, 1734, $1717 \mathrm{~cm}^{-1}$, was auf die Anwesenheit einer weiteren Hydroxylgruppe hinweist. Anschliessende Acetylierung von (II) mit Essigsäureanhydrid und Natriumacetate lieferte ein Triacetat (III) $\mathrm{C}_{26} \mathrm{H}_{40} \mathrm{O}_{6}$, Schmp. 195-198 , dessen IR-Spektrum keine Hydroxylbande mehr aufweist. Im NMR-Spektrum von (III) erscheinen neben den Signalen $(\delta=0.97,1.11,1.19$ und $1.58 \mathrm{ppm}$, je $3 \mathrm{H}$, jedes Singulett) der vier tertiären Methylgruppen und denen $(\delta=1.92$, 2.00 und $2.02 \mathrm{ppm}$ ) der drei Acetylgruppen die Signalen der Protonen (2H) an den Acetoxygruppen tragenden Kohlenstoffatomen bei $5.10 \mathrm{ppm}$ als komplexes Multiplett. Demnach stellt die Substanz C ein tetracyclisches Diterpenderivat mit zwei sekundären und einer tertiären Hydroxylgruppe dar.

Bei der Oxydation mit Chromtrioxyd-Pyridin-Komplex lieferte (I) ein Diketon (IV), $\mathrm{C}_{20} \mathrm{H}_{30} \mathrm{O}_{3}$, Schmp. 197-199 ${ }^{\circ}$, mit IR-Banden $3450 \mathrm{~cm}^{-1}(\mathrm{OH})$ und $1710 \mathrm{~cm}^{-1}$ (sechsgliedriges Ringketon). Im NMR-Spektrum sind die Signale von vier tertiär gebundenen Methylgruppen
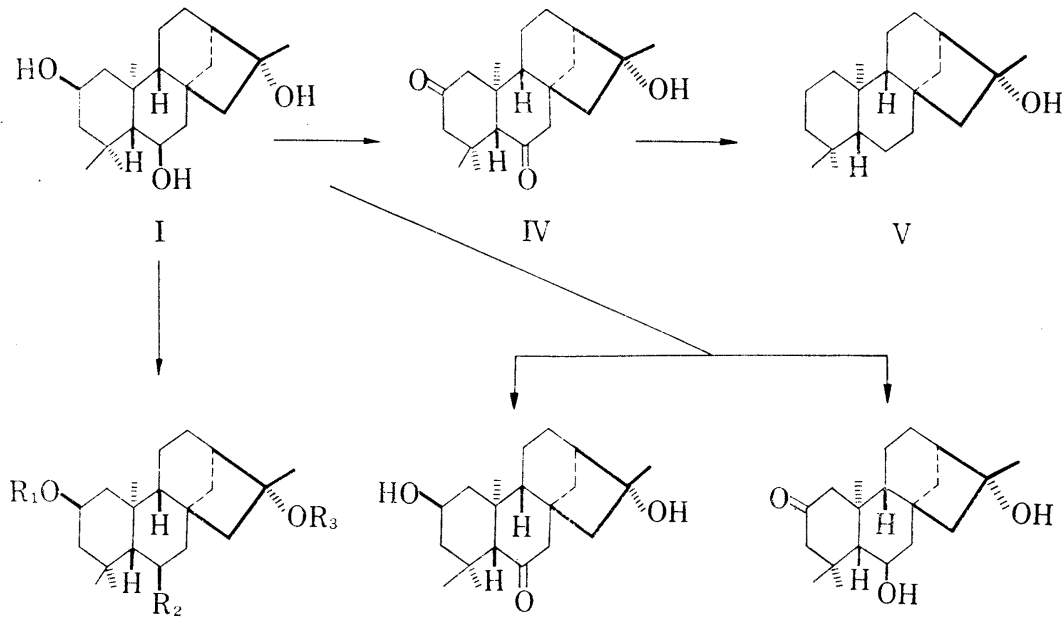

II $: \mathrm{R}_{1}=\mathrm{COCH}_{3}, \mathrm{R}_{2}=\mathrm{OCOCH}_{3}, \mathrm{R}_{3}=\mathrm{H} \quad \mathrm{VI}$

Lil : $\mathrm{R}_{1}=\mathrm{R}_{3}=\mathrm{COCH}_{3}, \mathrm{R}_{2}=\mathrm{OCOCH}_{3}$

VII

III : $\mathrm{R}_{1}=\mathrm{COCH}_{3}, \mathrm{R}_{2}=\mathrm{R}_{3}=\mathrm{H}$

1) C.I. Chen und T. Murakami, Tetrahedron Letters, 1971, 1121.

2) C.M. Chen und T. Murakami, Chem. Pharm. Bull. (Tokyo), 19, 1495 (1971).

3) Die NMR-Spektren (in $\mathrm{CDCl}_{3}$ ) wurden im JNM-4H-100 mit TMS als innerem Standard, die IR-Spektren (in $\mathrm{KBr}$ ) im $\mathrm{JASCO}-403 \mathrm{G}$ von Nihon Bunko aufgenommen. 
bei $1.04,1.10,1.25$ und $1.38 \mathrm{ppm}$ sichtbar. Das Singulett $(1 \mathrm{H})$ bei $2.73 \mathrm{ppm}$ ist der an eine Carbonylfunktion gebundenen Methingruppe zuzuordnen, die von zwei vollsubstituierten C-Atomen benachbart ist. Also kommt für die Stellung dieser Ketogruppe C-6- oder C-11Position in Betracht.

Bei der Huang-Minlon-Hydrierung von Diketon (IV) resultierte Monool (V), $\mathrm{C}_{20} \mathrm{H}_{34} \mathrm{O}$, Schmp. 208-210 $,[\alpha]_{\mathrm{D}}^{20}:-44^{\circ}\left(c=0.2, \mathrm{CHCl}_{3}\right)$. Diese Verbindung stimmte jetzt in allen chemischen und physikalischen Daten mit (-)-Kauranol überein.

Durch Oxydation von (I) mit Chromtrioxyd-Pyridin-Komplex in der Kälte (5 Stunden) wurden als Hauptprodukt ein Monoketon (VI), $\mathrm{C}_{20} \mathrm{H}_{32} \mathrm{O}_{3}$, Schmp. 206-209 mit IR-Banden $3350 \mathrm{~cm}^{-1}(\mathrm{OH})$ und $1710 \mathrm{~cm}^{-\mathbf{1}}(\mathrm{CO})$ und in kleiner Menge ein zweites Monoketon (VII), $\mathrm{C}_{20} \mathrm{H}_{32} \mathrm{O}_{3}$, Schmp. $187-189^{\circ}$ mit IR-Banden $3350 \mathrm{~cm}^{-1}(\mathrm{OH})$ und $1710 \mathrm{~cm}^{-1}(\mathrm{CO})$ erhalten. Im NMR-Spektrum (in Pyridin- $d_{5}$ ) von (VI) findet sich das Signal des Oxymethin-Protons bei $4.25 \mathrm{ppm}$ als Tripeltriplett $\left(J_{\mathrm{ax}-\mathrm{ax}}=11 \mathrm{~Hz}, J_{\mathrm{ax}-\mathrm{äq}}=3 \mathrm{~Hz}\right)$. Dies lässt bei (VI) auf die $\mathrm{C}$-2-äquatoriale und damit $\beta$-ständige sekundäre Hydroxylgruppe schliessen. Im NMRSpektrum von (II) verschiebt sich eines der beiden Singulette der C-4-geminalen Dimethylgruppen gegenüber dem entsprechenden Signal des $2 \beta$-Acetoxy, 16 $\alpha$-hydroxy-(-)-kaurans (2 $\beta$-Acetoxy-(-)-kauranols) (VIII) ${ }^{\mathbf{1}}$ nach tieferem Feld, während das Signal der C-10-Methylgruppe dabei praktisch unverändert bleibt (vgl. Tabelle). Dies weist bei (I) auf die C-6Stellung der zweiten sekundären Hydroxylgruppe hin. Weiterhin liegt in (VII) das Signal des Oxymethin-Protons bei $4.18 \mathrm{ppm}$ als Tripeldublett $\left(J_{\mathrm{ax}-\mathrm{ax}}=11 \mathrm{~Hz}, J_{\mathrm{ax}-\ddot{u} \mathrm{q}}=4 \mathrm{~Hz}\right)$, was für das Vorliegen der C-6-äquatorialen und somit $\beta$-ständigen Hydroxylgruppe spricht.

TABeLle. Chemische Verschiebungen ( $\delta$-Werte) der Methylgruppen an $\mathrm{C}-4$ und $\mathrm{C}-10$ (in $\mathrm{CDCl}_{3}$ )

\begin{tabular}{lcc}
\hline & C-4-Dimethyl & C-10-Methyl \\
\hline (-)-Kauranol & $0.81,0.85$ & 1.03 \\
VIII & $0.91,0.93$ & 1.14 \\
II & $0.95,1.08$ & 1.18 \\
\hline
\end{tabular}

Nach diesen Ergebnissen wird der Substanz C die Struktur 2 $\beta, 6 \beta, 16 \alpha$-Trihydroxy-(-)kauran (I) zugeordnet.

Faculty of Pharmaceutical Sciences, Science University of Tokyo Ichigaya Funakawaramachi, Shinjuku-ku, Tokyo

Eingangen am 21, September 1972
Chiu-Ming Chen

Takao Murakami 